\title{
Temperature dependence of deformation-assisted crystallization in amorphous $\mathrm{Fe}_{78} \mathrm{~B}_{13} \mathrm{Si}_{9}$
}

\author{
J. $\mathrm{Xu}^{\mathrm{a})}$ \\ Department of Nuclear Engineering and Radiological Sciences, The University of Michigan, Ann Arbor, \\ Michigan 48109-2104 \\ M. Atzmon ${ }^{\text {b) }}$ \\ Department of Nuclear Engineering and Radiological Sciences and Department of Materials Science and \\ Engineering, The University of Michigan, Ann Arbor, Michigan 48109-2104
}

(Received 27 May 1998; accepted for publication 24 July 1998)

\begin{abstract}
Low-energy ball milling of amorphous $\mathrm{Fe}_{78} \mathrm{~B}_{13} \mathrm{Si}_{9}$ has been performed at temperatures between 25 and $250{ }^{\circ} \mathrm{C}$. Primary crystallization is observed after milling at elevated temperatures, but not after annealing at the same temperatures. Although milling at room temperature creates nucleation sites for primary crystallization, subsequent annealing at elevated temperature does not result in significant crystallization. Heating by ball impact and contamination by the milling tools during milling are ruled out as the causes for crystallization. The observed behavior is interpreted to be caused by diffusivity enhancement due to milling-produced defects. (C) 1998 American Institute of Physics. [S0003-6951(98)01439-9]
\end{abstract}

During the past decade, formation of amorphous alloys by mechanical alloying (MA) of elemental powder mixtures $^{1,2}$ and/or by mechanical grinding of a single-phase intermetallic compound ${ }^{3,4}$ has been investigated extensively for a number of alloys. It has often been suggested that the disordering effect of deformation favors amorphous phases. ${ }^{5}$ Since atomic diffusion is potentially enhanced by nonequilibrium defect formation during deformation, a complete description of phase formation needs to take into account the competition between disordering and back-diffusion toward equilibrium (see, e.g., Ref. 6). In amorphous phases, if the latter dominates, milling-enhanced crystallization is expected.

Few authors have reported on crystallization of amorphous alloys during mechanical grinding. Trudeau et al. ${ }^{7,8}$ first reported that high-energy ball milling of Fe-based amorphous alloys caused their crystallization, a process referred to as mechanical crystallization. Bansal et al. ${ }^{9}$ argued that this observation was a result of impurity incorporation during milling, and not intrinsic to the deformation. Huang et al. ${ }^{10}$ reported mechanically induced crystallization during cryogenic ball milling of $\mathrm{Fe}_{78} \mathrm{~B}_{13} \mathrm{Si}_{9}$ metallic glass and suggested heating as a cause for enhanced crystallization kinetics. He et al. ${ }^{11}$ reported deformation-induced crystallization in several Al-based metallic glasses. The authors showed that nanocrystallites formed in shear bands induced by bending, and judged heating effects to be unlikely. Although the mechanism has not been explained in detail, observed compositional changes accompanying crystallization ${ }^{12}$ indicate long-range atomic interdiffusion. An unexplained cyclic transformation between the amorphous and crystalline state in ball-milled Co-Ti alloys has been reported by El Eskandarany et al. ${ }^{13}$ To date, a full understanding of mechanical

\footnotetext{
${ }^{a}$ Permanent address: Institute of Metal Research, Chinese Academy of Sciences, Shenyang, 110015, People's Republic of China.

${ }^{b)}$ Corresponding author. Electronic mail: atzmon@umich.edu
}

crystallization has not been reached, and the subject remains controversial. In order to reach a better understanding of the process, low-energy ball milling (LEBM) of amorphous $\mathrm{Fe}_{78} \mathrm{~B}_{13} \mathrm{Si}_{9}$ has been carried out at different temperatures, and the resulting transformations have been characterized by means of x-ray diffraction (XRD) and differential scanning calorimetry (DSC).

Amorphous $\mathrm{Fe}_{78} \mathrm{~B}_{13} \mathrm{Si}_{9}$ produced by Allied Metglas Products (trade name 2605-S2) was cut into pieces about $1 \mathrm{~cm}^{2}$ in size, $8 \mathrm{~g}$ of such material were sealed in a stainlesssteel vial with stainless-steel balls (4:1 ball-to-sample ratio) in an argon atmosphere and comminuted using a SPEX 8000 mixer/mill for $1 \mathrm{~h}$ to form flake-shaped particles. $5 \mathrm{~g}$ of the resulting powder were used as starting material for each subsequent anneal or LEBM run at room temperature, $200^{\circ} \mathrm{C}$, or $250^{\circ} \mathrm{C}$. LEBM was carried out in a vibrating-frame grinder (FRITSCH Pulverisette 0). The vial was evacuated to below $10^{-3}$ Torr using a sorption pump and backfilled with purified argon gas to a pressure above $150 \mathrm{kPa}$, kept by a continuous argon supply during milling. In contrast to high-energy mills, heating of the powder by ball impact in this grinder is estimated to be less than $1{ }^{\circ} \mathrm{C},{ }^{14}$ so that the temperature uncertainty is $\pm 1^{\circ} \mathrm{C}$. To minimize gaseous contamination, each sample was obtained in a continuous, uninterrupted run. Samples were annealed in a Ti-gettered inert-gas furnace after purging the atmosphere for an extended time.

XRD measurements were performed in a Rigaku diffractometer with monochromated $\mathrm{Cu} K \alpha$ radiation generated by a rotating-anode source. Thermal analysis was performed in a Perkin Elmer DSC-7 under flowing purified argon. A second run under identical conditions was used to determine the baseline after each run. The chemical composition was examined for several typical samples using inductively coupled plasma emission spectroscopy (ICPES).

In Fig. 1(a), representative examples of XRD patterns are displayed for initially amorphous samples after milling at $250{ }^{\circ} \mathrm{C}$ for different times. Note the gradual appearance of a 


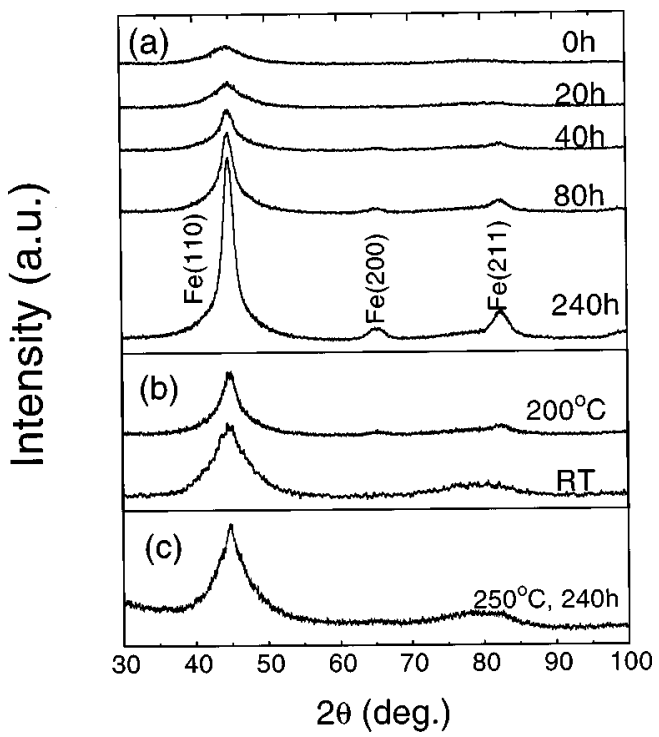

FIG. 1. XRD patterns for amorphous $\mathrm{Fe}_{78} \mathrm{~B}_{13} \mathrm{Si}_{9}$ after milling (a) at $250{ }^{\circ} \mathrm{C}$ for different times, (b) for $80 \mathrm{~h}$ at room temperature and $200^{\circ} \mathrm{C}$, (c) after annealing at $250{ }^{\circ} \mathrm{C}$ for $240 \mathrm{~h}$.

bcc phase, but no other crystalline phase. Similar results were also observed for milling at $200^{\circ} \mathrm{C}$. In contrast, complete thermal crystallization of this alloy results in the formation of $\alpha$-Fe(Si) and $\mathrm{Fe}_{2} \mathrm{~B} .{ }^{15}$ For comparison, diffraction patterns obtained after milling for $80 \mathrm{~h}$ at room temperature and $200^{\circ} \mathrm{C}$ are presented in Fig. 1(b), and after annealing for $240 \mathrm{~h}$ at $250^{\circ} \mathrm{C}$ in Fig. 1(c). The full width at half maximum (FWHM) of the most intense diffraction peak is displayed in Fig. 2 for alloys annealed or milled at different temperatures. Since this peak consists of a contribution from the amorphous phase and the (110) peak of the bcc phase, the FWHM is a qualitative measure of the amorphous fraction of the alloy. It is observed to decrease with time, at a rate which is observed to (a) increase with increasing temperature, and (b) be enhanced by milling as compared to annealing at the same temperature.

The initial sample and representative samples milled at room temperature and $250^{\circ} \mathrm{C}$ were analyzed by ICPES. Enrichment in $\mathrm{Fe}$, the main contaminant, is below the detection limit, $<0.1$ at. $\%$. This indicates that contamination by wear debris during LEBM is insignificant, unlike for high-energy ball milling ${ }^{9}$ (e.g., SPEX 8000). Therefore, Fe incorporation is ruled out as a cause for crystallization.

Figure 3 displays DSC scans for alloys milled at $250{ }^{\circ} \mathrm{C}$

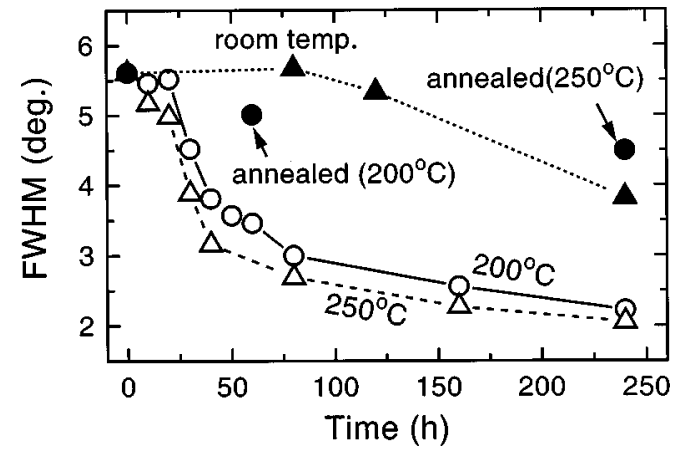

FIG. 2. FWHM of the most intense XRD peak as a function of time for different milling $(\triangle, \bigcirc, \boldsymbol{\Delta})$ or annealing $(\mathbf{O})$ temperatures.

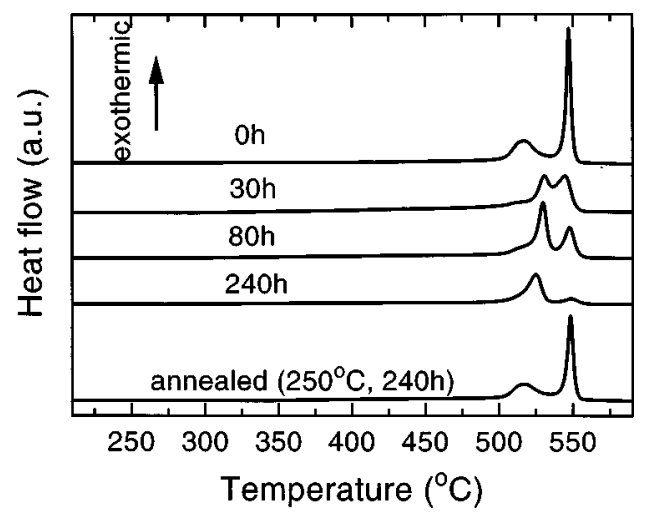

FIG. 3. DSC scans at $10{ }^{\circ} \mathrm{C} / \mathrm{min}$ for samples milled or annealed at $250^{\circ} \mathrm{C}$.

for different times or annealed at $250{ }^{\circ} \mathrm{C}$ for $240 \mathrm{~h}$. For all samples, three exothermic reactions are observed in the temperature range of $100-600^{\circ} \mathrm{C}$ at a heating rate of $10^{\circ} \mathrm{C} / \mathrm{min}$. A typical example is shown in Fig. 4, together with Pearsonfunction fits ${ }^{16}$ to the three peaks. The following transformations have been associated with similar peaks observed for the as-received alloy: ${ }^{15}$ a broad peak around $400{ }^{\circ} \mathrm{C}$ results from relaxation, and sharper peaks at 520 and $545^{\circ} \mathrm{C}$ result from primary crystallization of an Fe-rich bcc phase and transformation of the remaining amorphous matrix to $\alpha$ - $\mathrm{Fe}(\mathrm{Si})$ and $\mathrm{Fe}_{2} \mathrm{~B}$, respectively. In our milled samples, a combination of XRD and DSC indicates that the first two DSC peaks are associated with the formation of the primary bcc phase only. For samples milled at room temperature, the first peak constitutes as much as $55 \%$ of the total heat release. This suggests that milling introduces heterogeneous nuclei or nucleation sites, resulting in a lowering of the temperature at which primary crystallization takes place upon subsequent heating.

In Fig. 5, the total integrated heat release obtained from the DSC scans is displayed as a function of time for samples milled or annealed at different temperatures. The contribution to the signal by relaxation and grain growth in an initially present crystalline phase are known to be small, and the heat release is dominated by the transformation of the amorphous phase into the final crystalline product. The trends in Fig. 5 are similar to those displayed by the FWHM (Fig. 2). The short-time anomalous increase of the heat release at $200^{\circ} \mathrm{C}$ in Fig. 5 is unexplained, because its magnitude, $2.2 \mathrm{~kJ} / \mathrm{mole}$, cannot be accounted for by relaxation of milling-induced defects. We note that only the evolution of the low-temperature DSC peak depends on the milling tem-

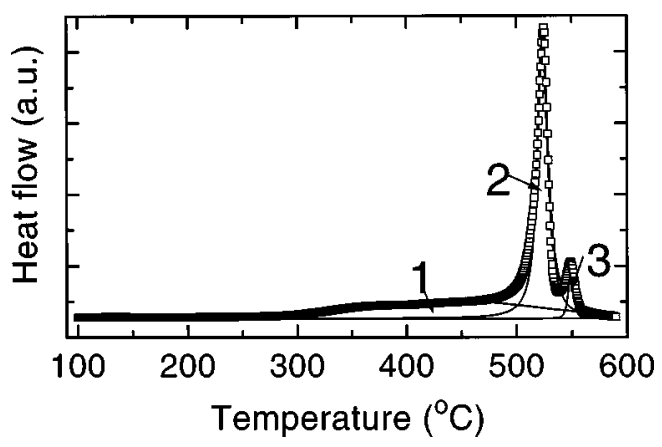

FIG. 4. Sample DSC scan with fits for sample milled for $240 \mathrm{~h}$ at $250^{\circ} \mathrm{C}$. 


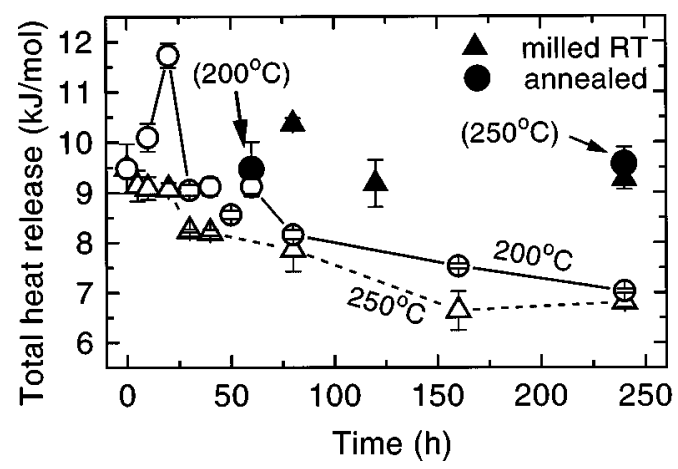

FIG. 5. Total heat release in DSC as a function of milling $(\triangle, \bigcirc, \boldsymbol{\Delta})$ or annealing (O) times at various temperatures.

perature. The temporal evolution of the area under the second and third DSC peaks is the same for all milling temperatures. The details of this behavior are the subject of further investigation.

One might suggest that the observed milling-enhanced crystallization at elevated temperature is due to conventional, thermal, growth at nucleation sites which have been created by deformation (see above). However, a sample milled $80 \mathrm{~h}$ at room temperature and subsequently annealed $60 \mathrm{~h}$ at $200^{\circ} \mathrm{C}$, displayed a FWHM of $5.3^{\circ}$ and total integrated heat release of $10.5 \mathrm{~kJ} / \mathrm{mole}$, significantly above the values for a sample milled $60 \mathrm{~h}$ at $200{ }^{\circ} \mathrm{C}$ [Figs. 2 and 5]. Thus, enhancement of crystallization requires simultaneous milling and annealing, suggesting that the enhancement is due to migration of milling-induced defects. While, in crystalline materials, vacancies are created by deformation and enhance the diffusivity, we suggest that the free volume in the amorphous phase plays a similar role. ${ }^{17}$ The microscopic details, however, are still unknown. The observed temperature dependence is consistent with analysis of defect production and annihilation kinetics. ${ }^{18}$ At room temperature, millinginduced defects are not mobile, whereas at higher temperatures, the temperature dependences of the steady-state defect diffusivity and defect annihilation rate cancel out so as to produce a weak temperature dependence of the effective diffusivity.

In summary, LEBM of amorphous $\mathrm{Fe}_{78} \mathrm{~B}_{13} \mathrm{Si}_{9}$ at elevated temperature under contamination-free conditions leads to partial crystallization of the amorphous phase to form primary $\alpha-\mathrm{Fe}(\mathrm{Si})$. Although room-temperature milling introduces nucleation sites, the crystallization rate is significant only when milling and annealing are simultaneous. The observed behavior is consistent with diffusion enhancement due to nonequilibrium point defects generated by plastic deformation.

This work was funded by the U.S. National Science Foundation, Grant No. DMR-9500617.

${ }^{1}$ C. C. Koch, O. B. Cavin, C. G. McKamey, and J. O. Scarborough, Appl. Phys. Lett. 43, 1017 (1983).

${ }^{2}$ L. Schultz, Mater. Sci. Eng. 97, 15 (1988).

${ }^{3}$ R. B. Schwarz and C. C. Koch, Appl. Phys. Lett. 49, 146 (1986).

${ }^{4}$ K. Yamada and C. C. Koch, J. Mater. Res. 8, 1317 (1993).

${ }^{5}$ H. Bakker, G. F. Zhou, and H. Yang, Mater. Sci. Forum 179-181, 47 (1995).

${ }^{6}$ G. Martin, Phys. Rev. B 30, 1424 (1984).

${ }^{7}$ M. L. Trudeau, R. Schulz, D. Dussault, and A. Van Neste, Phys. Rev. Lett. 64, 99 (1990).

${ }^{8}$ M. L. Trudeau, J. Y. Huot, R. Schulz, D. Dussault, A. Van Neste, and G. L'Esperance, Phys. Rev. B 45, 4626 (1992).

${ }^{9}$ C. Bansal, B. Fultz, and W. L. Johnson, Nanostruct. Mater. 4, 919 (1994).

${ }^{10}$ B. Huang, R. J. Perez, P. J. Crawford, A. A. Sharif, S. R. Nutt, and E. J. Lavernia, Nanostruct. Mater. 5, 545 (1995).

${ }^{11}$ Y. He, G. J. Shiflet, and S. J. Poon, Acta Metall. Mater. 43, 83 (1995).

${ }^{12}$ A. A. Csontos and G. J. Shiflet, Nanostruct. Mater. 9, 281 (1997).

${ }^{13}$ M. S. El Eskandarany, K. Aoki, K. Sumiyama, and K. Suzuki, Appl. Phys. Lett. 70, 1679 (1997); Scr. Mater. 36, 1001 (1997).

${ }^{14}$ Y. Chen, R. Le Hazif, and G. Martin, Diffus. Defect Data, Part B 23, 271 (1992).

${ }^{15}$ I. Matíko, E. Illekova, P. Svec, and P. Duhaj, Mater. Sci. Eng. A 225, 145 (1997); P. G. Caceres and K. H. Habib, Z. Metallkd. 87, 300 (1996).

${ }^{16}$ A. Brown and J. W. Edmonds, Adv. X-ray Anal. 23, 361 (1980).

${ }^{17}$ M. H. Cohen and D. Turnbull, J. Chem. Phys. 31, 1781 (1959).

${ }^{18}$ P. Pochet, E. Tominez, L. Chaffron, and G. Martin, Phys. Rev. B 52, 4006 (1995). 